\title{
Well-established monolingual literacy predictors in bilinguals
}

\author{
Insiya Bhalloo ${ }^{1,2}$, Kai Ian Leung ${ }^{1,2}$, Monika Molnar ${ }^{1,2}$ \\ ${ }^{1}$ Bilingual and Multilingual Development Lab, University of Toronto, Canada \\ ${ }^{2}$ Rehabilitation Sciences Institute, University of Toronto, Canada \\ https://doi.org/10.36505/ExLing-2020/11/0013/000428
}

\begin{abstract}
An important component of early reading intervention is effective literacy screening tools. Literacy precursor screening tools have been primarily developed for early identification and remediation of potential reading difficulties in monolingual Englishspeaking children, despite the significant proportion of bilingual children worldwide. This systematic literature review examines whether the precursor literacy skills commonly used in monolingual English-speaking children have been assessed and found to predict later reading skills in simultaneous bilingual children. Our findings demonstrate that the nine major literacy precursors identified in monolingual children also significantly correlate with reading performance in simultaneous bilingual children. These nine literacy precursors are phonological awareness, letter knowledge, serial recall, oral language comprehension, vocabulary, grammar, memory, non-verbal intelligence and word decoding.
\end{abstract}

Keywords: child literacy, education, literacy precursors, bilingualism, multilingualism

\section{Introduction}

Globally, reading difficulties are common in young children, with $56 \%$ of primary school-aged children unable to meet age-appropriate reading proficiency levels (UNESCO, 2017). Literacy precursors are language and cognitive skills, developed prior to a child receiving formal literacy instruction, that predict child success in future reading acquisition (Hjetland, Brinchmann, Scherer, \& Melby-Lervåg, 2017). Identifying literacy precursors is crucial for early intervention of children at risk for future word decoding and reading comprehension difficulties, as these children typically exhibit deficits in precursor literacy skills. Word decoding is the ability to first identify individual phonemes present in a written word and then sequence combinations of phoneme (sound) - grapheme (symbol) associations to pronounce/read a printed word, while reading comprehension is the ability to recognize and then understand written text. Based on previous systematic reviews conducted on monolingual literacy development (i.e. Hjetland et al., 2017), the following nine critical precursors of future word decoding and reading comprehension have been identified in monolingual children: (i) phonological awareness, (ii) letter knowledge, (iii) serial recall, (iv) oral language comprehension, (v) receptive and

ExLing 2020: Proceedings of $11^{\text {th }}$ International Conference of Experimental Linguistics, 12-14 October 2020, Athens, Greece 
expressive vocabulary, (vi) grammar (including morphological and syntactic awareness), (vii) memory, (viii) non-verbal intelligence, and (ix) word decoding.

The majority of child literacy development research has focused on monolingual children; however, approximately $50 \%$ of children worldwide grow up in a bilingual learning environment (e.g., Ryan, 2013). It is important to examine whether the literacy precursors, identified in monolingual children, have been assessed and found to predict reading development in bilingual children. As evident by previous research, differences have been observed in cognitive, speech, language and literacy development between monolingual and bilingual children (Thomas-Sunesson, Hakuta, \& Bialystok, 2018). Such a knowledge synthesis that focuses on bilingual children is therefore necessary in order to support reading abilities in both bilingual languages, due to these observed oral language and cognitive-linguistic differences.

\section{Purpose of the study}

This systematic review investigates whether the nine literacy precursor skills (as established in monolingual children) have been assessed and found to predict later reading performance, as measured by word decoding and reading comprehension, in one or both languages of simultaneous bilingual children.

Methods

We conducted a database search of the nine literacy precursor skills in relation to the two literacy outcome measures, word decoding and reading comprehension. We searched four linguistics and education-based electronic databases: Linguistics and Language Behavior Abstracts (LLBA), Educational Resources Information Center (ERIC), MLA International Bibliography and PsycINFO (ProQuest). The database search was conducted based on the PICO framework: Population (of typically-developing simultaneous bilingual children aged 12 years or younger), Intervention (the type of literacy precursor assessed in relation to reading), Comparison (not applicable, as we did not specify a mandatory monolingual, sequential bilingual or second language learner agematched comparison group) and Outcome (the type of reading-based literacy outcome measure assessed).

In our review, we only included peer-reviewed journal publications that assessed precursor literacy skills in relation to a reading-based literacy outcome measure in typically-developing simultaneous bilinguals (i.e. they were exposed to both languages before the age of 3 ) who were 12 years of age or younger. To account for the risk of bias, we excluded studies that did not meet the Critical Appraisal Checklist for Quasi-Experimental Studies (Joanna Briggs Institute, 2017). Additionally, we excluded studies that did not indicate sufficient child language background and parental socio-economic status information. 


\section{Results and discussion}

The database search resulted in a total of 2433 studies, with 1464 duplicate items. After screening for duplicates, 969 abstracts were identified as relevant for title and abstract-level screening. Two reviewers (IB and KIL) independently screened and identified 248 abstracts that met the inclusion criteria. After conducting the full-text screening and critical appraisal of these studies, 18 studies met the inclusion criteria. In addition, we manually searched the reference list of these 18 papers as well as the previously excluded nonprimary studies. Using Google Scholar, we also screened papers that cited these studies. This resulted in 22 additional studies (after the initial title and abstractlevel screening). After conducting full-text screening of these additional studies, 11 additional studies met the inclusion and critical appraisal criteria. In total, 29 studies (18 from the original full-text screening and 11 from the additional screening) met the inclusion and critical appraisal criteria and were included in this systematic literature review.

A total of 23/29 reviewed bilingual studies assessed these nine monolingual precursors in simultaneous bilingual children: phonological awareness (13 studies), letter knowledge (2 studies), serial recall (3 studies), oral language comprehension (3 studies), vocabulary (14 studies), grammar (6 studies), memory ( 2 studies), non-verbal intelligence (1 study), and word decoding (4 studies). The most commonly assessed literacy precursors in the reviewed bilingual studies were vocabulary, phonological awareness, grammar and word decoding. The literacy precursors with the greatest number of significant, as compared to non-significant, correlations were vocabulary, phonological awareness, oral language comprehension, letter knowledge and non-verbal intelligence (see Table 1).

The significant correlations between these nine literacy precursors and the literacy outcome measures indicate that similar literacy precursor screening tools and reading outcome measures, developed for monolingual children, may also be used for assessing simultaneous bilingual children. However, it is important that speech-language pathologists and educators do not generalize child literacy research findings on monolingual English-speaking children to monolingual and bilingual children from diverse language backgrounds. This review demonstrates that the specific type of literacy precursor assessed impacts the type and strength of within- and across-language correlations between given literacy precursors and literacy outcome measures. Further research on developing language-specific literacy precursor and literacy outcome measures, based on the language(s) of assessment's linguistic properties is needed to effectively assess child reading abilities and provide early reading intervention for monolingual and bilingual populations in all their spoken languages. Linguistic diversity in child literacy research and developing appropriate language-specific screening tools for various linguistic populations 
will enable early identification and intervention of potential reading difficulties in both heritage and societal languages.

Table 1. Significant and non-significant within- and across-language correlations between literacy precursors (indicated on the left) and literacy outcome measures in monolingual (Hjetland et al., 2017) and simultaneous bilingual children. Note. $\mathrm{M}=$ monolinguals; $\mathrm{SB}=$ simultaneous bilinguals; sig.= significant; N.S.= not-significant; $\mathrm{WL}=$ within-language; $\mathrm{AL}=$ across-language.

\begin{tabular}{|c|c|c|c|c|c|}
\hline Literacy Precursor & M & SB & & & \\
\hline & Sig. & $\begin{array}{l}\text { Sig. } \\
\text { WL }\end{array}$ & Sig. AL & $\begin{array}{l}\text { Sig. } \\
\text { WL \& AL }\end{array}$ & N.S \\
\hline Phonological Awareness $(\mathrm{n}=13)$ & $\mathrm{Y}$ & $\mathrm{n}=10$ & & $\mathrm{n}=3$ & \\
\hline Letter Knowledge ( $\mathrm{n}=2)$ & Y & $\mathrm{n}=1$ & & $\mathrm{n}=1$ & \\
\hline Serial Recall $(n=3)$ & Y & $\mathrm{n}=1$ & & $\mathrm{n}=1$ & $\mathrm{n}=1$ \\
\hline Oral Language Comprehension $(\mathrm{n}=3)$ & $\mathrm{Y}$ & $\mathrm{n}=1$ & & $\mathrm{n}=2$ & \\
\hline Vocabulary $(n=14)$ & Y & $\mathrm{n}=8$ & $\mathrm{n}=1$ & $\mathrm{n}=5$ & \\
\hline Grammar $(n=6)$ & $\mathrm{Y}$ & $\mathrm{n}=3$ & & $\mathrm{n}=2$ & $\mathrm{n}=1$ \\
\hline Memory $(n=2)$ & Y & & $\mathrm{n}=1$ & & $\mathrm{n}=1$ \\
\hline Non-Verbal Intelligence $(n=1)$ & $\mathrm{Y}$ & & & $\mathrm{n}=1$ & \\
\hline Word Decoding $(n=4)$ & Y & $\mathrm{n}=3$ & & & $\mathrm{n}=1$ \\
\hline
\end{tabular}

\section{References}

Hjetland, H. N., Brinchmann, E. I., Scherer, R., Melby-Lervåg, M. 2017. Preschool predictors of later reading comprehension ability: A systematic review. Campbell Systematic Reviews, 14, 1-156.

Joanna Briggs Institute. 2017. Critical appraisal checklist for quasi-experimental studies (non-randomized experimental studies).

Ryan, C. 2013. Language use in the United States: 2011. American Community Survey Reports, ACS-22. U.S. Census Bureau, Washington, DC.

Thomas-Sunesson, D., Hakuta, K., Bialystok, E. 2018. Degree of bilingualism modifies executive control in Hispanic children in the USA. International journal of Bilingual Education and Bilingualism, 21(2), 197-206.

United Nations Educational, Scientific and Cultural Organization (UNESCO). 2017. More than one-half of children and adolescents are not learning worldwide. 